Análisis organizacional de las pymes sector comercio de calzado zona centro Pasto. Organizational analysis of
pymes footwear industry trade
center area Pasto.

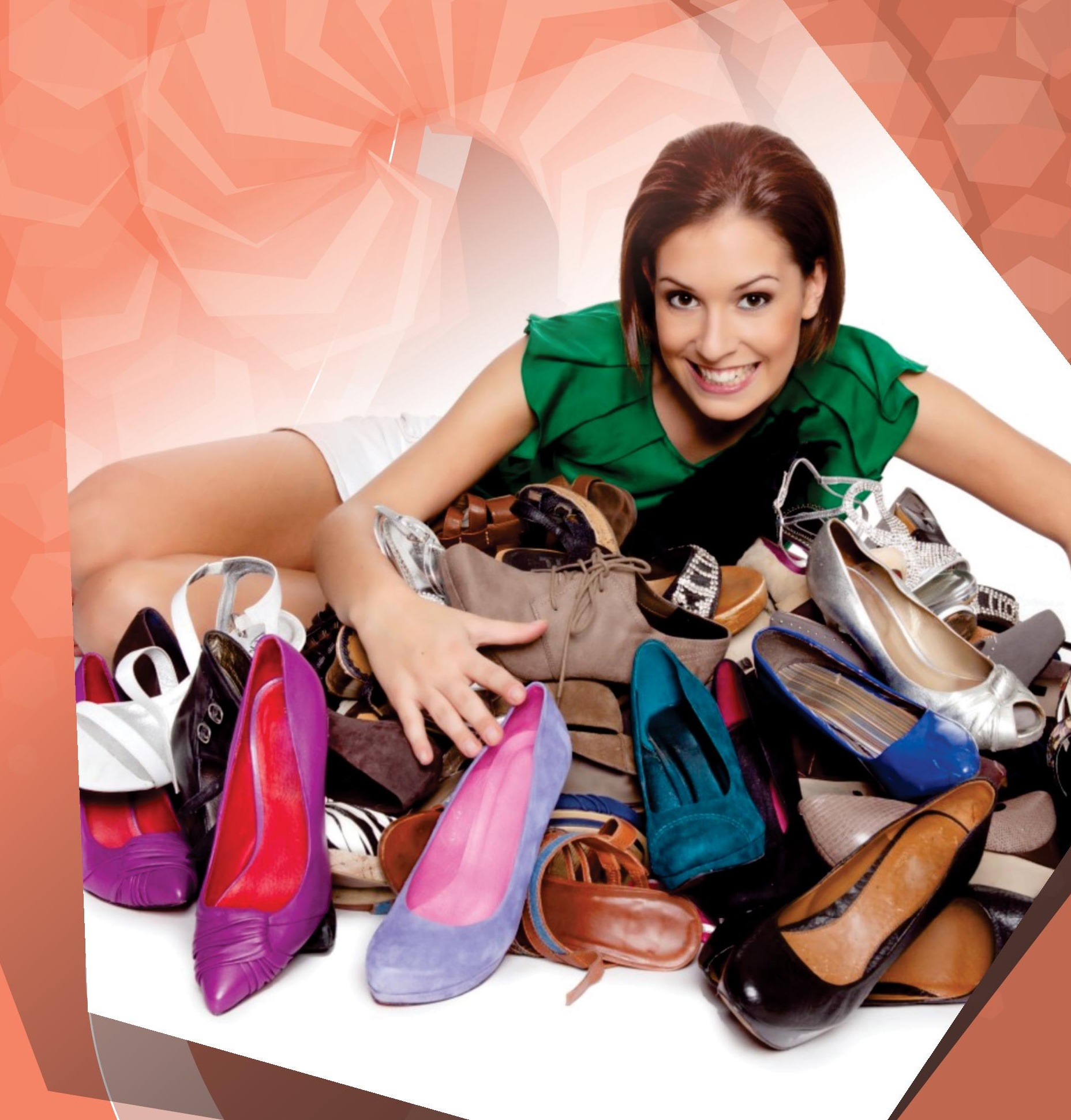




\title{
Análisis organizacional de las pymes sector comercio de calzado zona centro Pasto ${ }^{1}$ Organizational analysis of pymes footwear industry trade center area Pasto.
}

\author{
Silvio Augusto Delgado Unigarro ${ }^{2}$, Carlos Rubén Trejos Moncayo ${ }^{3}$ \\ Universidad Nacional Abierta y Distancia UNAD, CEAD Pasto, Colombia.
}

Artículo recibido en marzo de 2016; artículo aceptado en mayo de 2016

Citación del artículo: Delgado, S. \& Trejos, C. (2016). Análisis organizacional de las pymes sector comercio de calzado zona centro Pasto. I+D Revista de Investigaciones, 7(1), 98-108.

\section{Resumen \\ El presente artículo de investigación, orientado a establecer la situación organizacional como factor competitivo de las pymes dedicadas al comercio de calzado en la ciudad de San Juan de Pasto, muestra los resultados obtenidos a partir del trabajo de campo realizado con las mismas.}

Metodológicamente, la investigación se desarrolló estableciendo el marco teórico que sustentó las pretensiones de la investigación y que concretamente se enfatiza en la aplicación de las teorías del proceso administrativo y el análisis organizacional. La administración científica como base de teoría organizacional, la comprensión funcionalista, la teoría de la contingencia y la teoría de sistemas, recopiladas por Campos, Ferre, Martínez y Córdoba, entre otros, se constituyen como el principal referente teórico de la investigación.

Posteriormente, se desarrolló el diseño metodológico dirigido a obtener información relevante, mediante un trabajo de campo que consistió en la aplicación de una encuesta tipo Likert sometida a prueba de Alpha de Cronbach4 y que permitió la obtención de los resultados y el correspondiente análisis en donde se concluye que la forma cómo se está llevando la organización administrativa en una gran mayoría de las Pymes dedicadas al sector comercio de calzado en la ciudad de Pasto, presenta serias deficiencias, no garantiza la competitividad e inclusive, amenaza la existencia de algunas.

Palabras clave: Planeación, organización, dirección, control, análisis organizacional

\begin{abstract}
This research article indicates the results obtained from fieldwork with the SMEs, it engaged in the sale of footwear in the city of Pasto, aimed at establishing the organizational situation of the same as a competitive factor.

Methodologically at research the first step has been establish the Theoretical Framework that sustain the claims of the investigation and specifically emphasizes the application of theories of administrative process and organizational analysis. Then develops the methodological design, that seeks to obtain important information through a field work which included the application of a Likert survey subjected test for Alpha Cronbach and allowed to obtain results and the corresponding analysis, where it is concluded that the way is being the administrative organization in the vast majority of SMEs dealing in footwear sector in the city of Pasto, has serious deficiencies,
\end{abstract}

\footnotetext{
${ }^{1}$ Artículo de investigación, enfoque cuantitativo, resultado del proyecto de investigación "Nariño, un territorio por conocer" (culminado), perteneciente al área de Gestión Empresarial, sub área de Cultura Organizacional, desarrollado en el grupo de investigación SINDAMANOY y fue realizado con fondos de los investigadores. Fecha de inicio: Febrero de 2015, fecha de terminación: Enero de 2016.

${ }^{2}$ Administrador Público, ESAP. Maestría en Administración de las Organizaciones, UNAD. Docente- investigador del grupo: SINDAMANOY. Universidad UNAD de la ciudad de Pasto (Colombia): Carrera 21 ${ }^{\text {a }}$ No. 17-10, PBX: 7298520, Correo electrónico institucional: silvio.delgado@inad.edu.co

${ }^{3}$ Administrador de Empresas, UDENAR. Maestría en Mercadeo Agroindustrial, UDENAR. Docente- investigador del grupo: SINDAMANOY. Universidad UNAD de la ciudad de Pasto (Colombia): Carrera 21 ${ }^{\text {a }}$ No. 17-10, PBX: 7298520, Correo electrónico institucional: carlos.trejos@unad.edu.co

${ }^{4}$ El método de consistencia interna basado en el alfa de Cronbach permite estimar la fiabilidad de un instrumento de medida a través de un conjunto de ítems que se espera que midan el mismo constructo o dimensión teórica
} 
it does not guarantee the competitiveness and even threatens the existence of some.

Keywords: Planning, organization, managing, control, organizational analysis.

\section{Introducción}

Esta investigación busca, mediante la aplicación de los conceptos básicos de la teoría administrativa, encontrar explicaciones a situaciones puntuales del microentorno del subsector de calzado en la ciudad de Pasto. Siguiendo la propuesta de Rodríguez (2008), para cumplir con ese propósito, se realiza una revisión del mercado del calzado, se obtiene información directa que es muy importante ya que servirá como elemento constituyente para la formulación de estrategias de mejoramiento. De acuerdo con los objetivos de la investigación, su resultado permitirá encontrar y proponer soluciones concretas para una efectiva adopción de estrategias de tipo organizacional, fortaleciendo la dinámica gerencial en estos establecimientos comerciales, según las exigencias del sector.

En Pasto, la producción y comercialización de calzado ha sufrido una considerable afectación por diferentes factores como la mentalidad de producción artesanal, deficiencias en la organización del trabajo de la microempresa, falta de capital de trabajo e incapacidad de establecer estrategias que permitan afrontar las amenazas del medio externo. Sin embargo, es de destacar la gran habilidad y experiencia que el microempresario posee y, por lo tanto, es importante disponer de estrategias organizacionales con el fin de realizar una acción verdaderamente integral y más eficaz, tendientes a adecuar sus operaciones a los requerimientos del cliente, pensando en satisfacer sus necesidades y aprovechando las oportunidades que le ofrece el entorno en que se desarrolla. Al respecto, Ferre \& Ferre (1997) consideran que:

No solamente se hace necesario vivir en el mercado cada día y trabajar para, por y con él (filosofía de marketing). Sino que, además, la oferta de productos de toda empresa debe tratar de aportar "algo adicional" sobre lo que ofrece su competencia para ser claramente competitiva (p.8).

Las empresas objeto de estudio carecen de un monitoreo del mercado, lo cual no les permite tener información de primera mano, como investigaciones de mercado sobre tendencias de consumo, para llevar a cabo un proceso de toma de decisiones realmente efectivo. Con base en lo anterior, esta investigación aborda las unidades productivas objeto de estudio y presenta un acercamiento a la realidad que vive el sector, como un instrumento que aporte al mejoramiento de la situación actual.

El comercio del calzado en la ciudad de Pasto se ha visto amenazado especialmente por competidores extranjeros provenientes de Ecuador, China y Panamá quienes son más competitivos especialmente en términos de costos y frente a los cuales han estado perdiendo los mercados nacional y local, siendo estos esenciales para que esta actividad se pueda desarrollar y fortalecer lo suficiente como para afrontar los mercados y la competencia internacional con mayor seguridad. Delgado \& Torres (2012), mencionan que:

Ante la difícil situación, varios fabricantes han tratado de mantener su empresa a flote, el número de unidades productoras a nivel local ha disminuido en los últimos años, afectando tanto a pequeños empresarios, como a trabajadores directos e indirectos y demás actividades relacionadas con el subsector.

En el Departamento de Nariño se cuenta con la línea de redescuento en zona de frontera; este punto se constituye en una ventaja comparativa para las micro, pequeñas y medianas empresas de los departamentos ubicados en zonas de frontera.

La situación encontrada después de realizar el trabajo de campo, muestra que no hay una adecuada aplicación del proceso administrativo (planeación, organización, dirección y control) e integración con todas las actividades empresariales necesarias para eliminar la estrechez del mercado, además tiene que ver con distintas variables que afectan al subsector del calzado: información sobre el mercado, organización, formación técnica y administrativa de los empresarios, entre otras. De acuerdo a lo anterior, para que las pymes sean competitivas y por lo tanto mantengan y mejoren su posición en el mercado, se debe propender por la formulación de estrategias para que finalmente, los empresarios alcancen los resultados esperados.

El disponer de un análisis organizacional permitirá realizar una acción verdaderamente integral y más eficaz, con miras a adoptar efectivamente un enfoque competitivo, para lo cual es necesario un análisis de la situación que permita definir la ventaja competitiva y establecer la contribución que desde la academia se pueda ofrecer al sector.

Para lograr los objetivos propuestos, se acude al empleo de técnicas de investigación como las encuestas y su validación con información secundaria. La hipótesis se plantea a partir de la gestión administrativa de las microempresas, como un factor clave para su crecimiento y posicionamiento, desarrollando una cultura orientada hacia el mercado.

\section{Tipo de estudio}

\section{Método}

Dadas las pretensiones de la investigación y el fenómeno a analizar, la investigación fue de enfoque cuantitativo, descriptivo y transversal, no experimental. Con un método inductivo-deductivo.

Las variables que se abordan en el proceso investigativo son: formalización, competencias empresariales, 
direccionamiento estratégico y estructura organizacional. Hipótesis: con la correcta aplicación del proceso administrativo y el análisis organizacional, las pymes lograrán un incremento en la competitividad y por lo tanto, un mejoramiento de su posición en el mercado.

\section{Participantes}

El diseño muestral utilizado fue aleatorio simple, en tal sentido y teniendo en cuenta que se hizo un análisis organizacional de las pymes dedicadas al comercio de calzado de la zona centro Pasto, (las cuales se totalizan en 45 según registros de la Cámara de Comercio de Pasto) se consideró como muestra un total de 25 pymes. La muestra se establece según registro de la Cámara de Comercio de Pasto, donde el número de empresas caracterizadas para el estudio es reducido y por lo tanto, se permite el empleo de un margen de error más alto.

Como característica socio demográfica de la muestra, se rescata su trayectoria en el mercado, ubicación, tipo de organización y proyección frente a los cambios del entorno y del sector. (Direccionamiento estratégico y Planeación estratégica).

\section{Materiales e instrumentos}

Se estructuró una encuesta basada en una escala Likert como herramienta de recolección de información. Se sometió a una prueba piloto y se calculó el Alpha de Croanbach, con un resultado de 0.8 . Se midieron variables como: Formalización, reflejada en términos de legalidad de las pymes; Forma de constitución de las pymes (tipo de sociedad); Competencias empresariales, medidas a través del nivel académico del administrador o propietario; Profesión del administrador o propietario; Direccionamiento estratégico; Estructura organizacional (organigrama) que aborda aspectos tales como el conocimiento del proceso administrativo y mecanismos de selección de personal.

Para el proceso de tabulación, se utilizó una tabla de Excel en donde se determinó las frecuencias y porcentajes por cada variable y posteriormente, presentar los resultados orientados a la discusión final.

\section{Procedimiento}

Se visitó 25 pymes dedicadas a la comercialización de calzado, ubicadas en el sector centro de la ciudad de Pasto y se informó sobre las finalidades del presente proyecto de investigación, posteriormente se dio a conocer la estructura de la encuesta y, finalmente, se solicitó al administrador de la organización, proceder con el diligenciamiento de la encuesta, para lo cual se llevó a cabo un acompañamiento permanente para mantener la confiabilidad de la información suministrada. Simultáneamente, se realizó el proceso de observación directa sobre el objeto de estudio que permitió fortalecer el análisis de los resultados.

\section{Resultados}

Figura 1. Constitución de la Pyme

\section{Legalidad y constitución de la pyme}

$$
\text { MI PYME SE ENCUENTRA LEGALMENTE CONSTITUIDA. }
$$

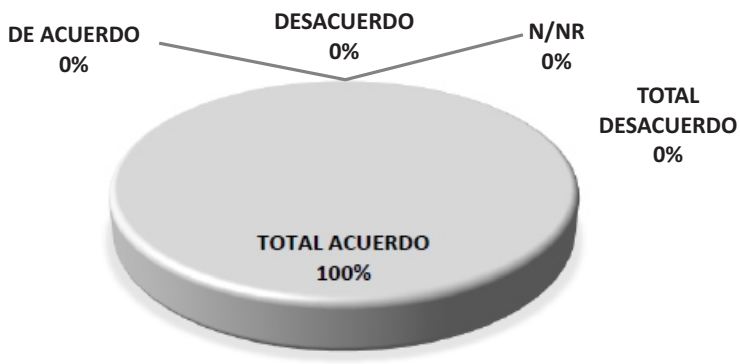

Fuente: Los autores

En Colombia las pymes son importantes en la economía. "La importancia de la estrategia, la estructura y las formas de asociación de las pequeñas y medianas empresas, representan una ventaja competitiva en el entorno" (Velásquez, 2004, p.1).

Siendo así, los indicadores económicos establecen que una parte considerable de las microempresas contribuyen al PIB. A diferencia del resto del país, en San Juan de Pasto, según los resultados anteriores, las empresas en su totalidad responden a la necesidad de establecerse legalmente ante las autoridades y ejercer libremente su actividad comercial. Este es un factor de confianza para las instituciones que basan su accionar en el acompañamiento empresarial como apoyo efectivo para el sector de calzado en la ciudad.

Tabla 1

Constitución como sociedad

\begin{tabular}{lccc}
\hline $\begin{array}{l}\text { Opción de } \\
\text { respuesta }\end{array}$ & $\begin{array}{c}\text { No. de } \\
\text { casos }\end{array}$ & Porcentaje & $\begin{array}{c}\% \\
\text { acumulado }\end{array}$ \\
\hline $\begin{array}{l}\text { Total } \\
\text { desacuerdo }\end{array}$ & 1 & $4,00 \%$ & $4,00 \%$ \\
$\begin{array}{l}\text { Desacuerdo } \\
\text { Acuerdo }\end{array}$ & 11 & $44,00 \%$ & $48,00 \%$ \\
Total acuerdo & 5 & $12,00 \%$ & $60,00 \%$ \\
NS/NR & 5 & $20,00 \%$ & $80,00 \%$ \\
Total & 25 & $20,00 \%$ & $100,00 \%$ \\
\hline
\end{tabular}

Fuente: Esta investigación

Lozano, (2010) analiza la importancia que tiene la asociatividad como una estrategia para afrontar los principales problemas que enfrentan las pymes, 
tales como el acceso a recursos financieros, la penetración en mercados locales e internacionales y la innovación y producción de nuevos productos.

En virtud de lo anterior, los empresarios reconocen la importancia que éste tema representa para la unidad productiva, pero los resultados denotan escasa asociatividad, característica propia de la región sur del País, las excepciones que se evidencian corresponden a casos unipersonales. Sería preciso intervenir en el gremio, profundizando sobre las ventajas de la asociatividad como estrategia competitiva y facilitando un acompañamiento efectivo para aprovechar las oportunidades que genera el entorno.

Figura 2. Perfil del administrador

\section{Competencias del administrador}

\begin{abstract}
EL ADMINISTRADOR O GERENTE ES PROFESIONAL EN CIENCIAS ADMINISTRATIVAS, CONTABLES ECONOMICAS O DE NEGOCIOS
\end{abstract}

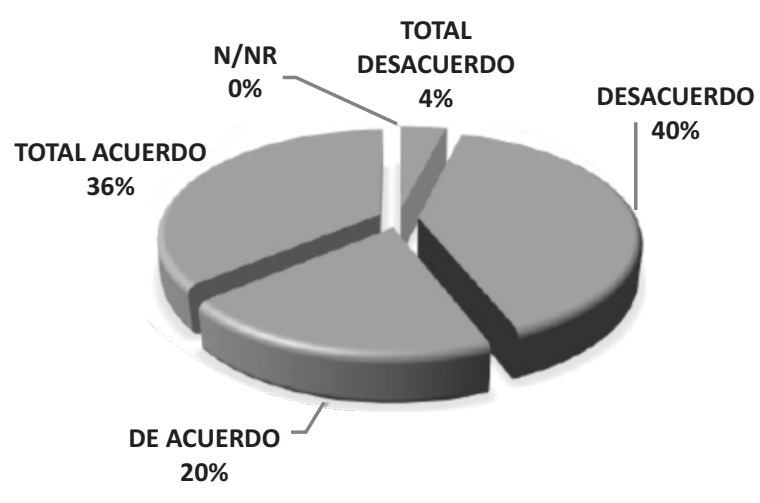

Fuente: Los autores

La figura 2 muestra elementos que refuerzan lo comentado por Giraldo, L \& Grisales, L. (2005) quienes identificaron las competencias que debe desarrollar un administrador, partiendo del interrogante sobre ¿qué características como personas (liderazgo, honestidad, ética, pedagogía, etc.), qué conocimientos en administración estratégica (planeación, organización, dirección y control) y qué destrezas (comunicación, negociación, interpretación de normas, delegación, etc.) se deben potencializar en los administradores?

De lo anterior se encontró una baja participación de profesionales en ciencias administrativas en la gerencia de estas organizaciones, priman otras profesiones en la administración de estas pymes que desconocen los procedimientos administrativos
Figura 3. Aplicación del proceso administrativo

EL GERENTE O ADMINISTRADOR APLICA EL PROCESO ADMINISTRATIVO (PLANEAR, ORGANIZAR, DIRIGIR Y CONTROLAR.

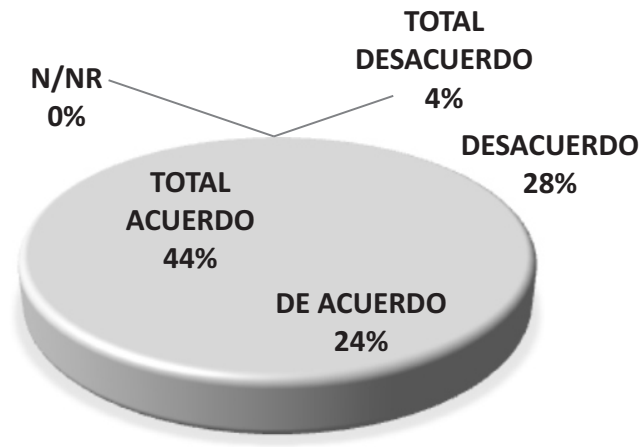

Fuente: Los autores

Siguiendo a Velásquez (2000) sobre el proceso administrativo de las organizaciones (las funciones de planeación, organización, dirección o influencia y control), desde la perspectiva del enfoque de sistemas y de contingencias, se evidencia que se aplica el proceso administrativo como función gerencial. La observación directa del trabajo de campo pudo identificar que en las pymes cuyo gerente es ajeno a las ciencias administrativas, este proceso se hace de forma empírica pues se tiene muchos años de trayectoria en el mercado y eso ha permitido desarrollar competencias frente al manejo administrativo de la organización.

\section{Direccionamiento Estratégico}

Figura 4. Misión, visión y objetivos

MI PYME TIENE ESTABLECIDO UNA MISIÓN, VISIÓN Y OBJETIVOS.

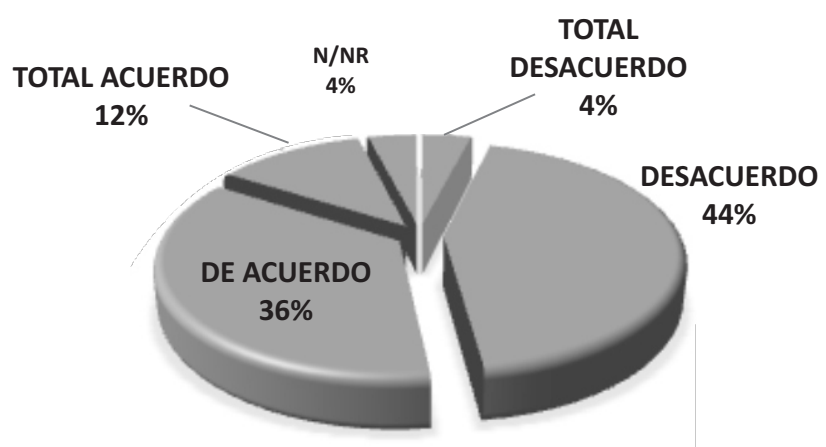

Fuente: Los autores

Aguilera, A. \& Riascos, S. (2009), afirman que:

La dirección estratégica debe ser un proceso continuo que apoye los objetivos a largo plazo trazados dentro de las empresas. Este proceso involucra la ejecución de un plan estratégico que sea reconocido, comprendido y aceptado por el personal encargado de ejecutarlo.

La aplicación del direccionamiento estratégico está 
dividido, es preciso fortalecer a estas organizaciones en la importancia de establecer una misión, visión y objetivos. Preocupa el desinterés de algunos gerentes que no consideran relevante tener un direccionamiento estratégico, pues no lo consideran relevante para su normal funcionamiento y dedican mayor interés a otros aspectos funcionales.

Figura 5: Plan de acción.

\section{EN MI PYME SE ESTABLECE UN PLAN DE ACCIÓN CADA AÑO.}

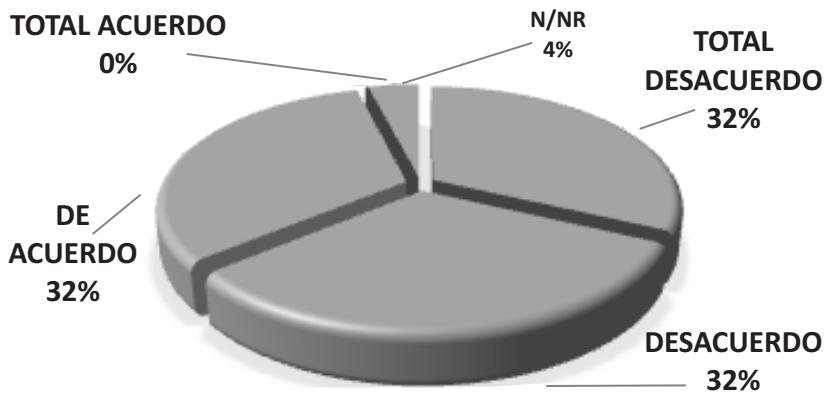

Fuente: Los autores

El plan de acción como hoja de ruta que permite orientar las acciones del negocio es ajeno a la mayoría de estas pymes, evidencia que no se ejerce la planeación como parte del proceso administrativo. Se proyecta todas las acciones a corto plazo y prima la inmediatez.

Si se analiza con detenimiento los resultados anteriores, la baja aplicabilidad de los planes de acción es un resultado de una ausencia de visión corporativa que ubique a la organización en escenarios futuros de crecimiento y permanencia. Aspecto que se consolida como una seria amenaza, en tiempos actuales de apertura económica y del desarrollo masivo de las TIC .

\section{Estructura Organizacional}

Figura 6. Existencia de Organigrama

EN MI PYME EXISTE UN ORGANIGRAMA ESTABLECIDO.

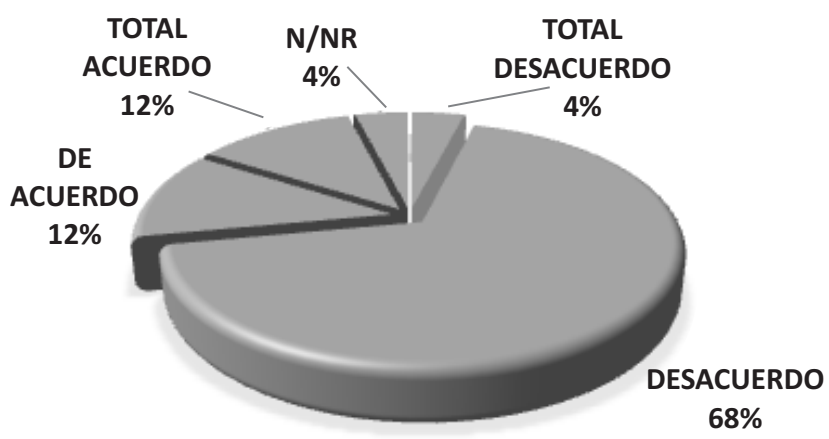

Fuente: Los autores
En 2012, Marín recoge los fundamentos teóricos acerca de los parámetros de diseño de la estructura de la organización, en el marco de la contingencia estructural, y da cuenta de la forma como las empresas están asumiendo eventos de re-estructuración. Sin embargo, en la investigación se denota manejo tradicional de las jerarquías y la departamentalización no existe.

Se desconoce la pertinencia de las estructuras organizacionales flexibles, como un factor diferenciador para alcanzar los objetivos y como ventaja competitiva, al momento de tener la capacidad de implementar estrategias que consoliden la organización en el sector.

\section{Herramientas de gestión}

Figura 7. Manual de funciones

EN MI PYME EXISTE UN MANUAL DE FUNCIONES.

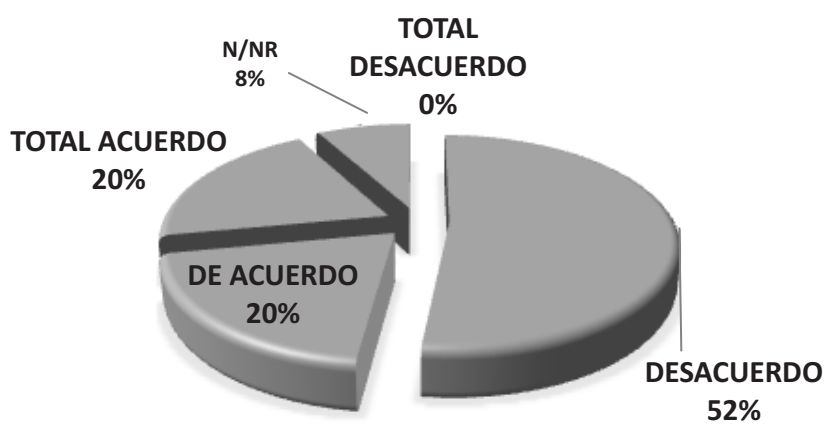

Fuente: Los autores

Las competencias comunicativas se consideran como uno de los aspectos fundamentales para la organización competitiva, por lo tanto, los gerentes y administradores deben velar por el desarrollo de estas competencias, mejorando sustancialmente el flujo de comunicación para el logro de los objetivos corporativos. En el trabajo realizado, se encontró que la asignación de las funciones a realizar se hace de manera verbal, no se estipulan en el contrato de trabajo; además, la baja aplicación del manual de funciones puede acarrear problemas legales.

Figura 8. Reglamento de trabajo visible a los trabajadores EN MI PYME EXISTE UN MANUAL DE FUNCIONES.

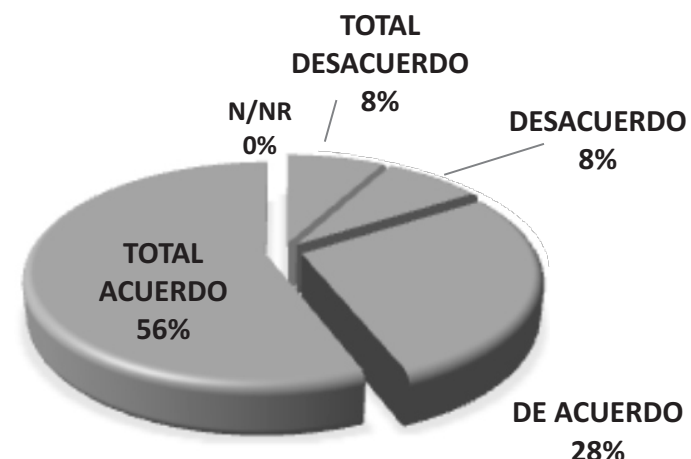

$28 \%$

Fuente: Los autores 
Por lo visto hasta el momento, las pymes tienen un alto grado de especialización en sus funciones y aunque no cuentan con algunos elementos importantes para el ejercicio empresarial efectivo, han dado respuesta efectiva a las demandas del mercado. Es así como se evidencia la existencia de un reglamento interno de trabajo visible al trabajador, situación positiva desde el punto de vista administrativo y legal.

Podemos inducir que existe la necesidad de identificar claramente los lineamientos para el desarrollo del trabajo, como un factor propio de la cultura organizacional de las empresas del sector.

Figura 9. Manual de procesos y procedimientos

EN MI PYME EXISTE UN MANUAL DE PROCESOS Y PROCEDIMIENTO.S

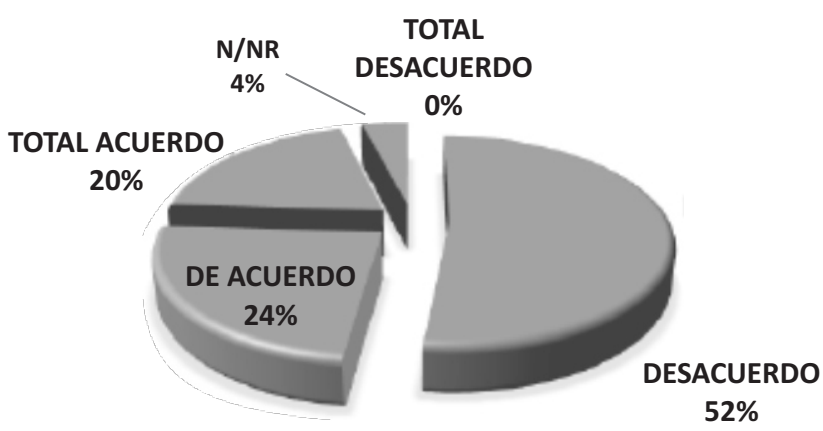

Fuente: Los autores

Para Medina, Noguera, Hernández y Díaz (2012), implementar un procedimiento de Gestión y Mejora de Procesos, dispone de mucha literatura y en su documento muestra una amplia revisión de procedimientos o metodologías, todas coincidentes con la necesidad de seleccionar los procesos a mejorar. Sin embargo, en el trabajo desarrollado, la asignación de procesos y procedimientos se hace de manera verbal y empírica, se enfatiza más en los procedimientos y se pudo constatar que se hace referencia a un manual de inventarios.

Figura 10. Proceso para la selección de personal

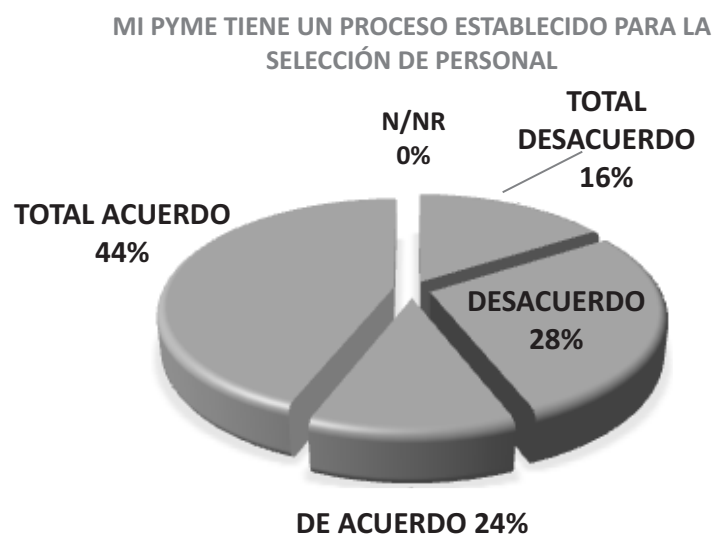

Fuente:Los autores
Aunque la mayoría de las empresas afirman tener establecido un manual para la selección de personal, en realidad, el proceso de observación adelantado por los investigadores evidenció que no lo tienen. El proceso de selección de personal se hace a través de empresas especializadas en este campo (outsourcing) o a través del SENA.

\section{Comentarios}

Sin duda alguna, es indiscutible la gran importancia que tienen las pequeñas y medianas empresas a la hora de fomentar el empleo, la innovación e instaurar ventajas competitivas en actividades de producción o prestación de servicio. De hecho, Páez como se citó en Acosta et al., (2004), las define como dispositivos socioeconómicos que generan riquezas y empleo. Por otra parte, el comercio representa grandes proporciones para enriquecer la productividad y competitividad en todos los niveles del desarrollo. Sin embargo, es primordial que las pymes establezcan condiciones adecuadas orientadas a la participación de la economía global sobre la base de asumir estrategias tecnológicas, organizacionales, productivas y legales. Al respecto, Anzola (2010) señala que: "Para continuar con el crecimiento y buen funcionamiento es necesario contar con conocimientos básicos sobre administración para poder, así, tomar las decisiones correctas". (Borbón, Amayrami y Espinoza, 2010, p.5)

La mercadotecnia de las pymes es una de las actividades de mayor importancia para el pequeño empresario y a la cual dedica gran parte de su tiempo en la empresa. Según Anzola citado por Borbón, Amayrami Espinoza (2010), generalmente, en las pymes el dueño funge también como gerente $\mathrm{y}$, por lo tanto, es el encargado de tomar las decisiones en cuanto a mercadotecnia, estas decisiones suelen estar basadas en corazonadas o en el gusto del empresario Sin esta actitud proactiva el futuro de las pymes a corto o mediano plazo será su desaparición del mercado.

Organizacionalmente hablando, los resultados obtenidos en esta investigación evidencian una clara tendencia a las deficiencias administrativas reflejadas por una parte, a la ausencia de profesionales en ciencias administrativas a cargo de la gerencia y por otro lado a la inaplicabilidad del proceso administrativo, el uso de herramientas gerenciales y, en general, a una administración con procedimientos empíricos.

En definitiva, el análisis de Gestión Administrativa es una herramienta de desarrollo empresarial, que permite evaluar el grado de eficiencia y eficacia con el cual, los recursos humanos están cumpliendo la planificación, la organización, la dirección, la coordinación, la ejecución y el control de los objetivos trazados por la alta gerencia. (Castro, 2007).

Algo similar se opina en la Revista para pymes (2007) en un artículo denominado Por qué muchas empresas fracasan: 
Es necesario abandonar la concepción de que sólo por hacer bien algo, podremos montar un negocio a su alrededor y conseguir llevarlo a buen puerto sin preocuparnos de conocer cómo funciona el mundo de gestión de empresa. Los pequeños y medianos negocios suelen sucumbir a causa de lo que se denominó "falta de educación empresarial", es decir, que no se conocían ni gestionaban bien los aspectos más básicos que mantienen vivo y hacen crecer un negocio. $\mathrm{Y}$ es que halando de educación empresarial y precisamente del sector comercio de calzado, Thomas Bata fundador de la más grande fábrica de calzado del mundo, atribuye el crecimiento explosivo de esa empresa al énfasis en la continua capacitación de su personal (León, 2010).

Ahora, para el Centro de Exportaciones e inversiones de Nicaragua (CEI, 2010) en la política de la liberalización de los mercados en los últimos años, han surgidos nuevos paradigmas para las organizaciones como la calidad total, la reingeniería y la mejora continua en el interior de las empresas tendientes a disminuir costos, aumento de la productividad, las alianzas estratégicas, las redes, la asociatividad, los clústeres y la colaboración de las cadenas productivas como formas de elevar la competitividad.

En esta investigación, las pymes estudiadas se encuentran en su totalidad registradas legalmente, la asociatividad es ajena a estas organizaciones, característica cultural del sur del País en donde prima el individualismo. "La asociatividad permite, a través de la implementación de estrategias colectivas y de carácter voluntario, alcanzar niveles de competitividad similares a los de empresas de mayor envergadura". (Liendo \& Martínez, 2001, p.5) Premisa que las pymes en cuestión deberían tener en cuenta.

Al mismo tiempo, toda organización sin importar el tamaño que proyecte cumplir los objetivos para los que fue creada, indiscutiblemente necesita contar y aplicar de forma eficiente las herramientas administrativas de tal manera que el conocimiento y ejerció del proceso administrativo es fundamental; Al respecto, "Ninguna organización puede alcanzar el éxito si no tiene una administración competente; es ella quien permite alcanzar las metas de las organizaciones, ya sean económicas, políticas o sociales, encauzando las aptitudes y energías humanas hacia una acción efectiva" (Márquez, \& Morela, 2002, p.11) Situación ajena para algunas de las pymes en cuestión que denotan en unos casos aplicabilidad empírica y en otros, ausencia total del proceso.

En concordancia, según (Córdova, 2012), la importancia del proceso administrativo se presenta porque es una guía práctica y metodológica para la administración, facilita sistematizar y controlar las operaciones, da las bases para estructurar el trabajo y dividirlo en departamentos de acuerdo a las necesidades y los recursos, permite establecer un sistema de selección de individuos de acuerdo a las necesidades, permite el desarrollo de capacidades y competencias laborales, facilita la dirección, al darle estrategia y control a quien la conduce y facilita el análisis de problemas para su solución. Es un importante instrumento de reorganización.

Con respecto a la existencia del reglamento interno de trabajo, (Mucenic citado por Quezada, 2010) manifiesta que:

El objetivo fundamental de este instrumento es el de lograr entre las partes que intervienen en la actividad de una empresa o establecimiento, una relación armónica y disciplinada que le permita a ésta ser realmente funcional y productiva con respecto al desarrollo de los trabajos realizados dentro de la organización.

Entonces, si retomamos la idea de que para las pymes es primordial establecer condiciones adecuadas de competitividad orientadas a la participación del mercado global, la condición legal es un factor importante en su actuar; en este sentido, prácticamente en 21 de las pymes analizadas señalan tener un reglamento interno visible al trabajador; situación positiva desde el punto de vista administrativo y legal, pero, preocupante para el 16\% de estas organizaciones cuyo reglamento no existe y por lo tanto, tendrán que comprender que es un instrumento importante para el establecimiento de las relaciones laborales.

El análisis organizacional de las pymes[U1] en el sector de calzado, en el centro de Pasto 2015, evidencia un bajo nivel de uso de las herramientas gerenciales como: manuales de funciones, procedimientos, y selección de personal. "Aunque el manual de funciones es bastante conocido, es evidente que por sí solo no tiene una aplicación práctica en la empresa es necesario combinar una serie de elementos fundamentales que hacen de su implementación un proceso exitoso" (Agudelo; Castañeda; Rojas, 2009, p.17). Es relevante para las pymes la adopción de este manual en pro de conocer mejor el funcionamiento interno en lo que respecta a la descripción de tareas, ubicación, requerimientos y puestos responsables de su ejecución. Por su parte, el manual de procedimientos ayuda a facilitar la supervisión del trabajo mediante la normalización de las actividades, evitando la duplicidad de funciones y los pasos innecesarios dentro de los procesos, agilizando la labor de la auditoria administrativa, la evaluación del control interno y su vigilancia (Flores, 2010); sin duda alguna una herramienta necesaria para unificar y controlar las rutinas de trabajo y evitar su alteración arbitraria dentro de las actividades de las pymes.

Como señala Espinosa (1965) en su conferencia Sin título, sobre la importancia de una buena administración en las empresas:

"La insuficiencia de los cuadros administrativos usualmente acarrea desperdicios, estrangulamientos de producción, un trato deficiente a la clientela, políticas inadecuadas de ventas y descontento entre el personal. Poco a poco estos factores comienzan a minar la existencia misma de la empresa, hasta que las utilidades se desploman." 
En consecuencia, la idea es que este tipo de empresas, señala el presidente del BID (Banco Interamericano de Desarrollo), se inserten en el proceso de globalización y pasen de ser empresas de subsistencia a comportarse como corporaciones, que lo son, con políticas de gobierno corporativo, que participen activamente en las estrategias de la Responsabilidad Social Empresarial y que a la vez formen parte del Pacto Global (de responsabilidad social corporativa), propuesto en 1999 por el entonces Secretario General de la ONU, Cofi Annan.(Innovación y empresa,2008)

Finalmente, se sienta este precedente para futuras investigaciones o intervenciones de la Academia en pro del mejoramiento de estas pequeñas y medianas empresas que indudablemente son importantes para el desarrollo de la región.

\section{Conclusiones}

La formalidad de las empresas del sector es un buen indicador de la confianza en las organizaciones reguladoras de la actividad comercial y, por lo tanto, del buen ejercicio comercial por parte de la administración. Sin embargo, se pudo comprobar que la asociatividad es incipiente y no se reconoce como una alternativa para enfrentar los nuevos retos del mercado, frente a competidores del orden nacional e internacional.

En consecuencia, las competencias demostradas por el administrador, no son las propias del ejercicio gerencial efectivo, pues no se aplica con el rigor necesario las técnicas del proceso administrativo. No obstante, es importante resaltar que las empresas han logrado permanecer en el mercado gracias a una acertada toma de decisiones por parte de sus gerentes que, en la mayoría de los casos, obedece a su buen juicio y criterio basados en la experiencia.

Uno de los puntos débiles encontrados, fue la ausencia de estrategias o lineamientos de mercadeo como resultado del proceso producto-mercado. Las empresas saben hacer bien su trabajo, pero descuidan un área de vital importancia cuando se trata de competir en un mercado altamente dinámico y de posicionarse en la mente de un consumidor más exigente y mejor informado.

Por su parte, el análisis organizacional de las pymes, del sector de calzado en el centro de Pasto en 2015, permitió determinar que existen marcadas deficiencias administrativas y organizacionales relacionadas al desconocimiento y a la inaplicabilidad del proceso administrativo, normatividad y uso de herramientas gerenciales, situación que no favorece la competitividad de estas organizaciones, por el contrario amenaza su existencia.

\section{Referencias}

Acosta, I; Pérez, M; Velazco, J. (2009). Las pymes en el desarrollo de la economía social. Factores de éxito subsector contratista del municipio lagunillas del Estado
Zulia. NEGOTIUM Revista Científica Electrónica Ciencias Gerenciales. Scientific e-journal of Management Science. Recuperado de:

http://www.revistanegotium.org.ve/pdf/12/Art6.pdf

Agudelo, A; Castañeda, P; Rojas, L. (2009). Diseño del manual de procesos, procedimientos y funciones para la distribuidora e importadora c. i coffee inn de la ciudad de Pereira (Risaralda). Recuperado de: http://es.slideshare.net/vasquezlr/manual-deprocedimiento-s-2

Aguilera, A. \& Riascos, S. (2009). Direccionamiento estratégico apoyado en las TIC. Estudios Gerenciales, 25(111), 127-143. Retrieved April 13, 2015, from http://www.scielo.org.co/scielo.php?script=sci_arttext\&pi $\mathrm{d}=\mathrm{S} 0123-59232009000200007 \& \operatorname{lng}=$ en\&tlng=es.

Borbón, R.; Amayrami, P.; Espinoza, F. (2010.). El impacto de la pequeña empresa en el desarrollo de la economía regional. Recuperado de: $\mathrm{http} / /$ revistainvestigacionacademicasinfrontera.com/files/ 12-1.pdf

Cáceres, G. Saavedra, S. y otros. (2005).Herramientas Gerenciales para una mejor administración ambiental aplicables a la Región Andina. Revista visión gerencial. $\mathrm{R}$ e c u p e r a d o d e : http://www.saber.ula.ve/bitstream/123456789/25068/2/arti culo2.pdf

Castro, Y. (2007). Diagnóstico de la gestión administrativa de la empresa NYC distribuciones, C.A., ubicada en la zona industrial III de Barquisimeto Estado Lara. Universidad Centro Occidental Lisandro Alvarado. República Bolivariana de Venezuela. Recuperado de: http://bibadm.ucla.edu.ve/edocs_baducla/tesis/p758.pdf

Campos, V. (2012). Teorías organizacionales postmodernas y la gestación del sujeto postmoderno. Departamento de Psicología de la Salut y de Psicología Social Universidad Autónoma de Barcelona. Recuperado e $\mathrm{n}$ : h t t p : / / w w w p s i u c v c l/ w p content/uploads/2012/11/vsc1de12.pdf

Centro de Exportaciones e Inversiones Nicaragua. (2010). Asociatividad. Recuperado de : http://www.cei.org.ni/images/file/manual_asociativ.pdf

Córdova, R. (2012). Proceso Administrativo. ISBN. Red tercer Milenio S.C.

Primera edición. Recuperado en: http:/www.aliatuniversidades.com.mx/bibliotecasdigitale s/pdf/sistemas/Proceso_administrativo/Proceso_administr ativo-Parte1.pdf

Delgado, C. \& Torres, J. (2012). Análisis y Caracterización del subsector calzado en el área metropolitana de Bucaramanga. Recuperado el 21 de 
octubre de 2014 en:

http://repositorio.uis.edu.co/jspui/bitstream/123456789/84 80/2/143208.pdf

Espinosa, M. (1965). Sin título, sobre la importancia de una buena administración en las empresas. Centro de estudios Espinoza Yglesias. Recuperado en: http://www.ceey.org.mx/sites/default/files/1965-18.pdf

Ferre, J. \& Ferre, J. (1997). Los Estudios de Mercado: Cómo Hacer un Estudio de Mercado de Forma Práctica. Todo lo Que Conviene Saber para Hacer Estudios con Escasos Recursos. Madrid. Ediciones Díaz de Santos.

Flores, A. (2010). Propuesta de manuales administrativos para la empresa Ferre Pat de Puebla, S. A. de $C$. Universidad veracruzana, facultad de contaduría y a d m i n i s t r a c i ó $\mathrm{n}$. R e c u p e r a d o d e : http://cdigital.uv.mx/bitstream/123456789/24168/1/Alicia MarFlores.pdf

Giraldo, L \& Grisales, L. (2005). Identificación y normalización de las competencias en los gerentes de las instituciones prestadoras de servicios de salud. Revista Facultad Nacional de Salud Pública, 23(2), 31-51. R e tri e ved A pril 13,2015 , from http://www.scielo.org.co/scielo.php?script=sci_arttext\&pi $\mathrm{d}=\mathrm{S} 0120$-386X2005000200005\&lng=en\&tlng=es

Hurtado, H. (2011). Modelos de gestión para pymes. Universidad nacional Abierta y a Distancia UNAD, Palmira. Disponible en:

http://datateca.unad.edu.co/contenidos/107027/Document os_pymes/Modelos_de_gestion.pdf

Instituto Nacional de la Administración pública (1997).La innovación y el análisis organizacional, Buenos Aires. Disponible en:

http://www.sgp.gov.ar/contenidos/inap/publicaciones/docs /capacitacion/organi2.pdf

Innovación y empresa, (2008). Los múltiples retos de las pymes en América Latina. Publicado el 28 de mayo. Recuperado de:

http://www.wharton.universia.net/index.cfm?fa=viewArti cle\&ID $=1527$

León, R. (2010).Importancia de la Capacitación del Personal de la Capacitación del Personal en las Utilidades de la Empresa en las Utilidades de la empresa. LEON \& Asociados. Consultoría y Capacitación Empresarial. Recuperado de:

http://www.gerenciayempresa.com/documentos/articulos/ IMPORTANCIA_DE_LA_CAPACITACION.pdf

Liendo, M. \& Martínez, A. (2001). Asociatividad. Una alternativa para el desarrollo y crecimiento de las pymes. Instituto de Investigaciones Económicas, Escuela de Economía. Universidad nacional del Rosario. Recuperado de: http://docplayer.es/11117367-Asociatividad-unaalternativa-para-el-desarrollo-y-crecimiento-de-laspymes.html

Lozano, M. (2010). Modelos de asociatividad: estrategias efectivas para el desarrollo de las Pymes. Revista $E A N$, (68), 175-178. Retrieved April 13, 2015, from http://www.scielo.org.co/scielo.php?script=sci_arttext\&pi $\mathrm{d}=\mathrm{S} 0120-81602010000100014 \& \operatorname{lng}=$ en\&tlng=es.

Marín, D. (2012). Estructura organizacional y sus parámetros de diseño: análisis descriptivo en pymes industriales de Bogotá. Estudios Gerenciales, 28(123), 4364. Recuperado en 13 de abril de 2015, de http://www.scielo.org.co/scielo.php?script=sci_arttext\&pi $\mathrm{d}=\mathrm{S} 0123-59232012000200004 \& \operatorname{lng}=\mathrm{es} \& \operatorname{tlng}=\mathrm{e}$.

Márquez, \& Morela. (2002). La gestión administrativa de las empresas agropecuarias de los municipios san Fernando y Biruaca del Estado Apure, en Venezuela. Revista Mexicana de Agronegocios. vol. VI, núm. 10. Sociedad Mexicana de Administración Agropecuaria A.C. Torreón, México.

Medina, A; Noguera, D; Hernández, A y Díaz, Y. Consideraciones y criterios para la selección de procesos para la mejora: Procesos Diana. Ing. Ind. [online]. 2012, vol.33, n.3 [citado 2015-04-14], pp. 272-281. Disponible en:

http://scielo.sld.cu/scielo.php?script=sci_arttext\&pid=S18 15-59362012000300007\&lng=es\&nrm $=\overline{\text { iso }}$

Plata, L. (2009, Noviembre, 24) Subsidios para pymes ubicadas en zonas de frontera. Revista Dinero. Recuperado de: http://www.dinero.com/comercio-exterior/edicionimpresa/articulo/subsidios-para-pymes-ubicadas-zonasfrontera/87199

Quezada, K. (2010). La importancia del Reglamento Interior de Trabajo. Kreston internacional. Recuperado de: http://www.duransilva.com.mx/webdocs/La\%20importanc ia $\% 20$ del $\% 20$ Reglamento $\% 20$ Interior $\% 20 \mathrm{de} \% 20$ T rabajo. pdf

Revista Recursos Para Pymes. (2007). Por qué muchas empresas fracasan. R e cuperado d e : https://utecno.files.wordpress.com/2013/06/fracasopymes.pdf

Rodríguez, A. (2008). La comparación de casos en el análisis organizacional: cronología de un trabajo de campo. Documentos y aportes en administración pública y gestión estatal, ( 11 ), 63-88. Recuperado de: http://www.redalyc.org/articulo.oa?id=337530216003

Sánchez, A. \& Serna, N. (2007). Las necesidades de las pyme -pequeñas y medianas empresas y el sistema de información contable y financiero como una estrategia para atenderlas. R e cuperado d e : 
http://revistas.javeriana.edu.co/index.php/cuacont/article/ view/3232

Shild, P. (2010). Importancia del Reglamento Interno de Trabajo en su Empresa. Recuperado de: http://exitojuridico.blogspot.com/2010/04/importanciadel-reglamento-interno-de.html

Velásquez, F. (2004). La estrategia, la estructura y las formas de asociación: fuentes de ventaja competitiva para las pymes colombianas. Estudios Gerenciales, 20 (93), 7397. Retrieved A pril 13, 2015 , from http://www.scielo.org.co/scielo.php?script=sci_arttext\&pi $\mathrm{d}=\mathrm{S} 0123-59232004000400003 \& \operatorname{lng}=\mathrm{en} \& \operatorname{tlng}=\mathrm{es}$
Velásquez, F. (2000). El enfoque de sistemas y de contingencias aplicado al proceso administrativo. Estudios Gerenciales, 16(77), 27-40. Retrieved April 13, 2015, from http://www.scielo.org.co/scielo.php?script=sci_arttext\&pi $\mathrm{d}=\mathrm{S} 0123-59232000000400002 \& \operatorname{lng}=$ en\&tlng=es 